PROCEEDINGS OF THE

AMERICAN MATHEMATICAL SOCIETY

Volume 125, Number 4, April 1997, Pages 971-977

S 0002-9939(97)03747-7

\title{
RINGS WITH FINITE ESSENTIAL SOCLE
}

\author{
JOSÉ L. GÓMEZ PARDO AND PEDRO A. GUIL ASENSIO
}

(Communicated by Ken Goodearl)

\begin{abstract}
Let $R$ be a ring such that every direct summand of the injective envelope $E=E\left(R_{R}\right)$ has an essential finitely generated projective submodule. We show that, if the cardinal of the set of isomorphism classes of simple right $R$-modules is no larger than that of the isomorphism classes of minimal right ideals, then $R_{R}$ cogenerates the simple right $R$-modules and has finite essential socle. This extends Osofsky's theorem which asserts that a right injective cogenerator ring has finite essential right socle. It follows from our result that if $R_{R}$ is a CS cogenerator, then $R_{R}$ is already an injective cogenerator and, more generally, that if $R_{R}$ is CS and cogenerates the simple right $R$-modules, then it has finite essential socle. We show with an example that in the latter case $R_{R}$ need not be an injective cogenerator.
\end{abstract}

\section{INTRODUCTION}

In her paper [9] Osofsky proved that a right injective cogenerator ring $R$ (i.e., a right $\mathrm{PF}$ ring) has finite essential right socle and is, therefore, semiperfect. This deep result has been used by several authors, particularly in questions related to the FGF problem which asks whether a ring $R$ such that every finitely generated right $R$-module embeds in a free module must be quasi-Frobenius (see e.g. [1, 5, 8, 6]). The purpose of this paper is to extend Osofsky's theorem by showing that under rather more general hypotheses on $R$ one can combine Osofsky's powerful counting argument with module-theoretic techniques, based on the connections that exist between $R$ and the endomorphism ring of its injective envelope, to obtain that $R$ has finite essential right socle.

Thus we replace the injectivity of $R_{R}$ by the much weaker condition that every direct summand of the injective envelope $E\left(R_{R}\right)$ has an essential finitely generated projective submodule, and we replace the cogenerating condition by the fact that the number of isomorphism classes of simple right $R$-modules is bounded by the number of homogeneous components of $\operatorname{Soc}\left(R_{R}\right)$, and we show that $R_{R}$ still has finite essential socle. The first of these conditions is satisfied by all the right CS rings (rings $R$ such that every complement right ideal is a direct summand of $R_{R}$ [2], also called right extending rings, cf. [3]) and so we obtain as an immediate corollary that every right CS ring $R$ such that $R_{R}$ cogenerates the simple right

Received by the editors September 28, 1995.

1991 Mathematics Subject Classification. Primary 16L30; Secondary 16D50, 16E50, 16L60, $16 \mathrm{~S} 50$.

This work was partially supported by the DGICYT (PB93-0515, Spain). The first author was also partially supported by the European Community (Contract CHRX-CT93-0091) and the Xunta de Galicia (XUGA 10502B94), and the second author by the C. A. de Murcia (PIB 94-25). 
$R$-modules has finite essential right socle. It should be remarked, however, that this class of rings is strictly larger than that of right PF rings, as is shown by some examples. Another consequence of our main result is a different extension of Osofsky's theorem: a ring $R$ such that $R_{R}$ is CS and a cogenerator is right PF. We also obtain some other applications and, in particular, we show that a right CS ring such that every cyclic right module embeds in a free module is right artinian.

Throughout this paper, all rings $R$ will be associative and with identity, and Mod- $R$ will denote the category of right $R$-modules. By a module we will usually mean a right $R$-module and, whenever we want to emphasize the fact that $M$ is a right $R$-module, we will write $M_{R}$. We refer to $[4,12]$ for all undefined notions used in the text.

\section{Results}

We begin by recalling from [6] the definition of an idempotent-orthogonal family of simple modules, and by introducing a more general concept that will be useful later on.

Definition 2.1. Let $S$ be a ring and $\left\{C_{k}\right\}_{k \in K}$ a family of pairwise nonisomorphic simple right $S$-modules. The family is said to be an idempotent-orthogonal family of simple modules (cf. [6]) when there exists a family $\left\{e_{k}\right\}_{k \in K}$ of idempotents of $S$ satisfying that $C_{j} e_{k}=0$ if $j \neq k$, and $C_{k} e_{k} \neq 0$ for each $k \in K$. More generally, the family will be called idempotent-semiorthogonal when $C_{k} e_{k} \neq 0$ for each $k \in K$ and, if $j \neq k$, then either $C_{j} e_{k}=0$ or $C_{k} e_{j}=0$.

The next lemma is the result of applying Osofsky's counting argument in [9] to our more general situation, and it is an immediate consequence of [6, Lemma 2.1, Lemma 2.3]. We denote by $|X|$ the cardinality of a set $X$.

Lemma 2.2. Let $R$ be a ring, $E_{R}$ an injective module, $S=\operatorname{End}\left(E_{R}\right)$ and $J=$ $J(S)$. Let $\left\{E_{i}\right\}_{i \in I}$ be a set of representatives of the isomorphism classes of indecomposable direct summands of $E_{R}$. Then there exists a bijection between $I$ and the set of isomorphism classes of minimal right ideals of $S / J$. Moreover, if $|I|$ is infinite, there exists an idempotent-orthogonal family $\left\{C_{k}\right\}_{k \in K}$ of simple right $S / J$-modules such that $|I|<|K|$.

Lemma 2.3. Let $P_{R}$ be a finitely generated projective module, $E=E\left(P_{R}\right), S=$ $\operatorname{End}\left(E_{R}\right)$ and $J=J(S)$. If $N$ is a right ideal of $S$ containing $J$, then $N=\{s \in$ $S \mid s(P) \subseteq N E\}$.

Proof. It is clear that if $s \in N$, then $s(P) \subseteq N E$. To prove the converse inclusion, let $s \in S$ be such that $s(P) \subseteq N E$. Since $s(P)$ is finitely generated, there exist elements $h_{1}, \ldots, h_{n} \in N$ such that $s(P) \subseteq \sum_{i=1}^{n} \operatorname{Im} h_{i}$. Denote by $\pi_{i}: E^{n} \rightarrow E$ the canonical projections and set $h=\sum_{i=1}^{n}\left(h_{i} \circ \pi_{i}\right): E^{n} \rightarrow E$. Then $\operatorname{Im} h=\sum_{i=1}^{n} \operatorname{Im} h_{i}$ and hence $s(P) \subseteq \operatorname{Im} h$. Thus, if we set $X=\operatorname{Im} h$, with canonical projection $\beta: E^{n} \rightarrow X$ the canonical injection $\alpha: X \rightarrow E$, and $u: P \rightarrow E$ is the canonical inclusion, we obtain a morphism $f: P \rightarrow X$ such that $s \circ u=\alpha \circ f$. Since $P$ is projective, there exists a morphism $g: P \rightarrow E^{n}$ such that $\beta \circ g=f$. Using the injectivity of $E^{n}$ we also obtain an extension $t: E \rightarrow E^{n}$ of $g$, so that $t \circ u=g$. Thus we have

$$
s \circ u=\alpha \circ f=\alpha \circ \beta \circ g=h \circ g=h \circ t \circ u
$$


so that $(s-h \circ t) \circ u=0$ and hence $\operatorname{Ker}(s-h \circ t)$ is essential in $E_{R}$. This entails that $s-h \circ t \in J \subseteq N$ and, since $h \circ t=\sum_{i=1}^{n}\left(h_{i} \circ \pi_{i}\right) \circ t=\sum_{i=1}^{n} h_{i} \circ\left(\pi_{i} \circ t\right) \in N$, we see that $s \in N$.

If $R$ is a ring, $\Omega(R)$ will denote a set of representatives of the isomorphism classes of simple right $R$-modules. If $M$ is a right $R$-module, then $C(M)$ denotes a set of representatives of the isomorphism classes of simple submodules of $M$ (see [6]). We have:

Lemma 2.4. Let $R$ be a ring and $P_{R}$ a finitely generated projective module such that each direct summand of $E=E\left(P_{R}\right)$ has an essential finitely generated projective submodule. Let $S=\operatorname{End}\left(E_{R}\right)$ and $J=J(S)$. If $\left\{C_{k}\right\}_{k \in K}$ is an idempotentsemiorthogonal family of simple right $S / J$-modules, then there exists an injective mapping from $K$ to $\Omega(R)$.

Proof. Since idempotents of $S / J$ lift modulo $J$, there exist idempotents $\left\{s_{k}\right\}_{k \in K}$ of $S$ such that $C_{k} s_{k} \neq 0$ for any $k \in K$ and either $C_{j} s_{k}=0$ or $C_{k} s_{j}=0$ for $k \neq j$. Let $c_{k} \in C_{k}$ be an element such that $c_{k} s_{k} \neq 0$ for each $k \in K$, and let $p_{k}: S_{S} \rightarrow C_{k}$ be the homomorphism defined by $p_{k}(1)=c_{k} s_{k}$. If $s_{k *}=\operatorname{Hom}_{R}\left(E, s_{k}\right)$ is the endomorphism of $S_{S}$ given by left multiplication with $s_{k}$, we have that $\left(p_{k} \circ s_{k *}\right)(1)=c_{k} s_{k}^{2}=c_{k} s_{k}=p_{k}(1)$, and so $p_{k} \circ s_{k *}=p_{k}$, from which it follows that

$$
\left(p_{k} \otimes_{S} E\right) \circ s_{k}=\left(p_{k} \otimes_{S} E\right) \circ\left(s_{k *} \otimes_{S} E\right)=\left(p_{k} \circ s_{k *}\right) \otimes_{S} E=p_{k} \otimes_{S} E .
$$

If we set $E_{k}=s_{k}(E)$ and $E_{k}^{\prime}=\left(1-s_{k}\right)(E)$, then $E_{k}$ and $E_{k}^{\prime}$ have finitely generated projective essential submodules $P_{k}$ and $P_{k}^{\prime}$, respectively, so that $P_{k} \oplus P_{k}^{\prime}$ is essential in $E=E_{k} \oplus E_{k}^{\prime}$. Then $\left(p_{k} \otimes_{S} E\right)\left(P_{k}^{\prime}\right) \subseteq\left(\left(p_{k} \otimes_{S} E\right) \circ s_{k} \circ\left(1-s_{k}\right)\right)(E)=0$ and so, if $\left(p_{k} \otimes_{S} E\right)\left(P_{k}\right)=0$ we have that $\left(p_{k} \otimes_{S} E\right)\left(P_{k} \oplus P_{k}^{\prime}\right)=0$. Let $N_{k}=\operatorname{Ker} p_{k}$. Since $C_{k}$ is simple we have that $J \subseteq N_{k}$. Moreover, $\operatorname{Ker}\left(p_{k} \otimes_{S} E\right)=N_{k} E$ and so $P_{k} \oplus P_{k}^{\prime} \subseteq N_{k} E$. Thus we obtain from Lemma 2.3 that $1_{S} \in N_{k}$, a contradiction. This proves that $\left(p_{k} \otimes_{S} E\right)\left(P_{k}\right) \neq 0$. Let $h_{k}: P_{k} \rightarrow E_{k}, i_{k}: E_{k} \rightarrow E$, and $t_{k}=i_{k} \circ h_{k}: P_{k} \rightarrow E$ be the inclusions, and set $L_{k}:=\operatorname{Im}\left(\left(p_{k} \otimes_{S} E\right) \circ t_{k}\right)$, with canonical projection $q_{k}: P_{k} \rightarrow L_{k}$ and inclusion $w_{k}: L_{k} \rightarrow C_{k} \otimes_{S} E$. As we have just seen, $L_{k}$ is a (finitely generated) nonzero module, and hence we can choose for each $k \in K$ a simple quotient $U_{k}$ of $L_{k}$ with canonical projection $\pi_{k}: L_{k} \rightarrow U_{k}$. Denoting by $[X]$ the isomorphism class of a right $R$-module $X$, we define a map from $K$ to the set of isomorphism classes of simple right $R$-modules by assigning $k \mapsto\left[U_{k}\right]$.

To complete the proof of the lemma, we show that the map just defined is injective. Suppose that $\left[U_{j}\right]=\left[U_{k}\right]$ for $j, k \in K$. Since $\left\{C_{k}\right\}_{k \in K}$ is an idempotentsemiorthogonal family of simple right $S / J$-modules, we can assume that, say, $C_{k} s_{j}$ $=0$. Let $\varphi: U_{j} \rightarrow U_{k}$ be an isomorphism. If $\alpha_{k}: U_{k} \rightarrow E\left(U_{k}\right)$ denotes the inclusion, for each $k \in K$, we obtain by injectivity an $R$-homomorphism $\phi: E\left(U_{j}\right) \rightarrow E\left(U_{k}\right)$ satisfying that $\phi \circ \alpha_{j}=\alpha_{k} \circ \varphi$. Also, $\alpha_{k} \circ \pi_{k}$ has an extension $\pi_{k}^{\prime}$ to $C_{k} \otimes_{S} E$, so that $\alpha_{k} \circ \pi_{k}=\pi_{k}^{\prime} \circ w_{k}$. On the other hand, using the projectivity of $P_{j}$, we obtain a homomorphism $\psi: P_{j} \rightarrow P_{k}$ such that $\pi_{k} \circ q_{k} \circ \psi=\varphi \circ \pi_{j} \circ q_{j}$. Now, from the injectivity of $E$, we obtain an endomorphism $\tau: E \rightarrow E$, i.e., an element $\tau \in S$, such that $\tau \circ t_{j}=t_{k} \circ \psi$. Observe then that $\phi \circ \alpha_{j}=\alpha_{k} \circ \varphi$ is a monomorphism, and hence the morphism $\phi \circ \alpha_{j} \circ \pi_{j} \circ q_{j}: P_{j} \rightarrow E\left(U_{k}\right)$ is nonzero (with image isomorphic 
to $U_{j}$ ). Thus we see that:

$$
\begin{gathered}
0 \neq \phi \circ \alpha_{j} \circ \pi_{j} \circ q_{j}=\alpha_{k} \circ \varphi \circ \pi_{j} \circ q_{j}=\alpha_{k} \circ \pi_{k} \circ q_{k} \circ \psi=\pi_{k}^{\prime} \circ w_{k} \circ q_{k} \circ \psi= \\
\pi_{k}^{\prime} \circ\left(p_{k} \otimes_{S} E\right) \circ t_{k} \circ \psi=\pi_{k}^{\prime} \circ\left(p_{k} \otimes_{S} E\right) \circ \tau \circ t_{j}=\pi_{k}^{\prime} \circ\left(p_{k} \otimes_{S} E\right) \circ \tau \circ i_{j} \circ h_{j} .
\end{gathered}
$$

Assume now that $j \neq k$ and consider the homomorphism $p_{k} \circ \tau_{*} \circ i_{j_{*}}: s_{j} S \rightarrow C_{k}$, where $\tau_{*}=\operatorname{Hom}_{R}(E, \tau)$ and $i_{j_{*}}=\operatorname{Hom}_{R}\left(E, i_{j}\right)$. If we set $x:=\left(p_{k} \circ \tau_{*}\right)(1) \in C_{k}$, we have that $\left(p_{k} \circ \tau_{*} \circ i_{j_{*}}\right)\left(s_{j}\right)=\left(p_{k} \circ \tau_{*}\right)\left(s_{j}\right)=x s_{j} \in C_{k} s_{j}=0$. Tensoring with ${ }_{S} E$ we then see that $\left(p_{k} \otimes_{S} E\right) \circ\left(\tau_{*} \otimes_{S} E\right) \circ\left(i_{j_{*}} \otimes_{S} E\right)=0$ and, since $\tau_{*} \otimes_{S} E \cong \tau$ and $i_{j_{*}} \otimes_{S} E \cong i_{j}$, that $\left(p_{k} \otimes_{S} E\right) \circ \tau \circ i_{j}=0$, which gives a contradiction and shows that we must have $j=k$.

Theorem 2.5. Let $R$ be a ring and $P_{R}$ a finitely generated projective module such that each direct summand of $E=E\left(P_{R}\right)$ has an essential finitely generated projective submodule and $|\Omega(R)| \leq|C(P)|$. Then $P_{R}$ cogenerates the simple right $R$-modules and has finite essential socle.

Proof. Let $\mathcal{S}$ be the set of isomorphism classes of minimal right ideals of $S / J$. Since by Lemma 2.2 they correspond bijectively to indecomposable direct summands of $E_{R}$ we have that $|C(P)| \leq|\mathcal{S}|$. If $|\mathcal{S}|$ is infinite, again by Lemma 2.2 there exists an idempotent-orthogonal family $\left\{C_{k}\right\}_{k \in K}$ of simple right $S / J$-modules such that $|\mathcal{S}|<|K|$ and by Lemma 2.4 we have that $|K| \leq|\Omega(R)|$. Thus we obtain a chain of inequalities:

$$
|\Omega(R)| \leq|C(P)| \leq|\mathcal{S}|<|K| \leq|\Omega(R)|
$$

which show that $|\mathcal{S}|$ is finite, say $r$, and that $|\Omega(R)|=n \leq r$ is also finite. Thus we have that $\Omega(R)=C(P)$ and hence $P_{R}$ cogenerates the simple right $R$-modules.

Now let $C_{1}, \ldots, C_{r}$ be representatives of the elements of $\mathcal{S}$ and suppose that there exists a simple right $S$-module $C=C_{r+1}$ which is not isomorphic to any of the $C_{1}, \ldots, C_{r}$. For each $i=1, \ldots, r$ there exist idempotent elements $e_{1}, e_{2}, \ldots, e_{r} \in S$ such that, if $\bar{e}_{i}=e_{i}+J$, then $C_{i}=\bar{e}_{i}(S / J)$. Using the isomorphism $X \bar{e}_{i} \cong$ $\operatorname{Hom}_{S / J}\left(\bar{e}_{i}(S / J), X\right)($ for $X \in \operatorname{Mod}-S / J)$, we see that $\bar{e}_{i}(S / J) \bar{e}_{j}=0$ for $i, j \leq r, i \neq$ $j$, and $\bar{e}_{i}(S / J) \bar{e}_{i} \neq 0$ for all $i=1, \ldots, r$. Moreover, $C \bar{e}_{i} \cong \operatorname{Hom}_{S / J}\left(\bar{e}_{i}(S / J), C\right)=0$ for all $i=1, \ldots, r$. Thus the family of simple right $S / J$-modules $\left\{C_{i}\right\}_{i=1, \ldots, r+1}$ is idempotent-semiorthogonal with respect to the idempotents $\left\{\bar{e}_{1}, \ldots, \bar{e}_{r}, 1\right\}$. From Lemma 2.4 we obtain that $r+1 \leq n$, a contradiction that shows that the simple module $C$ cannot exist, and hence that $S / J$ is a semisimple ring. Therefore, $S$ is semiperfect and $E_{R}$ is finite-dimensional, so that $E_{R}$ is a finite direct sum of indecomposable submodules. From the preceding argument it also follows that $r \leq$ $n$ and hence that $r=n$. Since the number of isomorphism classes of indecomposable direct summands of $E_{R}$ is exactly $n$, we see that each of them is an injective envelope of a simple right $R$-module, so that $E_{R}$, and hence $P_{R}$, has finite essential socle.

Corollary 2.6. Let $R$ be a right semihereditary right finite-dimensional ring such that $|\Omega(R)| \leq|C(R)|$. Then $R$ is semisimple.

Proof. Since $R$ is finite-dimensional, each right ideal of $R$ contains an essential finitely generated right ideal, which is projective because $R$ is right semihereditary. Thus the hypotheses of Theorem 2.5 hold, and we see that every simple right $R$ module is isomorphic to a right ideal of $R$, and hence projective. 
In relation with the preceding corollary we remark that, as is well known, a right hereditary ring is right finite-dimensional if and only if it is right noetherian. However, the class of right semihereditary right finite-dimensional rings is substantially larger. For instance, it includes the right semihereditary right Ore domains, and also the right serial right nonsingular rings as was shown by Warfield [13, Theorem 4.1].

It is clear that if $R$ is a right CS (or right extending) ring, then every direct summand of $E\left(R_{R}\right)$ has an essential finitely generated projective submodule. Thus we obtain:

Corollary 2.7. Let $R$ be a ring such that $R_{R}$ is $C S$ and cogenerates the simple right $R$-modules. Then $R_{R}$ has finite essential socle.

If we strengthen a bit the hypotheses of the preceding corollary, we obtain the following characterization of right PF rings.

Corollary 2.8. Let $R$ be a ring. Then the following conditions are equivalent:

(i) $R$ is right $P F$.

(ii) $R_{R}$ is a CS cogenerator.

(iii) $R_{R}$ is a cogenerator, and every direct summand of $E\left(R_{R}\right)$ contains an essential finitely generated projective submodule.

Proof. (i) $\rightarrow$ (ii) and (ii) $\rightarrow$ (iii) are clear.

(iii) $\rightarrow$ (i) Using Theorem 2.5 we see that $R$ has finite essential right socle. Since $R_{R}$ is a cogenerator, it is well known that $R$ is then right PF.

Since a ring $R$ such that each cyclic right $R$-module has finite essential socle is right artinian, from Corollary 2.7 we immediately obtain:

Corollary 2.9. Let $R$ be a right $C S$ ring such that every cyclic right $R$-module embeds in a free module. Then $R$ is right artinian.

Observe that, using Theorem 2.5, a more general version of the preceding corollary (and also of Corollary 2.7) can be obtained by replacing the CS condition by the hypothesis that every direct summand of $E\left(R_{R}\right)$ contains an essential finitely generated projective submodule.

A ring $R$ is said to be right FGF (cf. [5]) whenever every finitely generated right $R$-module embeds in a free (or, equivalently, in a projective) module. The problem of whether a right FGF ring is QF is still open, but some positive results have been obtained recently in [6] and [10]. Since a right artinian right FGF ring is QF [5], we obtain from Theorem 2.5 the following corollary that extends [6, Theorem 3.7].

Corollary 2.10. Let $R$ be a right FGF ring such that every direct summand of $E\left(R_{R}\right)$ contains an essential finitely generated projective submodule. Then $R$ is $Q F$.

Examples 2.11. (i) In Theorem 2.5, the hypothesis that each direct summand of $E\left(P_{R}\right)$ has an essential finitely generated projective submodule cannot be replaced by its weaker form obtained by dropping the finite-generation condition. Let $F$ be a field, $F_{n}=F$ for each $n \in \mathbb{N}$, and $Q=\prod_{\mathbb{N}} F_{n}$. Then the subring $R=$ $F \cdot 1+\bigoplus_{\mathbb{N}} F_{n}$ of $Q$ (consisting of the eventually constant sequences of elements of $F)$ is a von Neumann regular ring with $\operatorname{Soc}(R)=\bigoplus_{\mathbb{N}} F_{n}$. Since every ideal of $R$ is countably generated, $R$ is hereditary (see, e.g., [7, Corollary 2.15]) and so each direct summand of $E(R)$ contains a (not necessarily finitely generated) essential 
projective submodule. On the other hand, if $C$ is a simple $R$-module which is not isomorphic to a minimal ideal of $R$, then $C$ is not projective and hence it is a singular module. This implies that $C \cong R / L$ for an essential proper ideal $L$ of $R$ and hence $\operatorname{Soc}(R) \subseteq L$. It is easily checked that $\operatorname{Soc}(R)$ is a maximal ideal of $R$ and so we must have $L=\operatorname{Soc}(R)$, which shows that there is just one isomorphism class of nonprojective simple $R$-modules. Thus we see that $|\Omega(R)|=|C(R)|\left(=\aleph_{0}\right)$ but $R / \operatorname{Soc}(R)$ does not embed in $R$ and $\operatorname{Soc}(R)$ is not finitely generated. This example also shows that the finite-dimensionality hypothesis cannot be dropped from Corollary 2.6.

(ii) As we have remarked, if $R$ is a right CS ring, then every direct summand of $E\left(R_{R}\right)$ contains an essential finitely generated projective submodule. However, the converse does not hold as is shown e.g. by the non-CS prime right hereditary right noetherian ring given in [2, Example 6.9]. In fact, there exists a (two-sided) artinian ring $R$ such that both $E\left(R_{R}\right)$ and $E\left({ }_{R} R\right)$ are projective but $R$ is not right CS. Let $R$ be the ring of upper triangular $2 \times 2$ matrices over $\mathbb{Z} / p^{2} \mathbb{Z}$, where $p$ is a rational prime. It is readily checked that the right ideal $\left\{\left(\begin{array}{cc}x & y \\ 0 & p y\end{array}\right) \mid x \in p \mathbb{Z} / p^{2} \mathbb{Z}, y \in \mathbb{Z} / p^{2} \mathbb{Z}\right\}$ is (essentially) closed but not a direct summand of $R_{R}$, so that $R$ is not right CS. On the other hand, let $A$ be the full $2 \times 2$ matrix ring over $\mathbb{Z} / p^{2} \mathbb{Z}$. It is easy to see that ${ }_{R} A$ is isomorphic to a direct summand of ${ }_{R} R \oplus_{R} R$, so that ${ }_{R} A$ is projective (and similarly, $A_{R}$ is projective). Moreover, it is clear that $A \otimes_{R}(A / R)=0$ and using e.g. [12, Proposition XI.1.2] we see that the inclusion of $R$ in $A$ is an epimorphism of rings. Now, since $\mathbb{Z} / p^{2} \mathbb{Z}$ is a self-injective ring, $A$ is right self-injective. Thus it follows from [12, Proposition XI.3.11] that $A_{R}$ is injective. It is then clear that $E\left(R_{R}\right) \cong A_{R}$ and hence $E\left(R_{R}\right)$ is projective.

(iii) The ring of upper triangular $2 \times 2$ matrices over a field $F$ is right CS and has finite essential socle (as it is right artinian) but it does not cogenerate all the simple right modules, so that the converse of Corollary 2.7 does not hold. This highlights the interest of Corollary 2.8, in view of the well-known fact that if $R$ is right self-injective, semiperfect with essential right socle, then $R$ is right $\mathrm{PF}$.

(iv) Let $F\left[X_{1}, X_{2}, \ldots\right]$ be the polynomial ring on a countable set of commuting indeterminates $X_{1}, X_{2}, \ldots$ over a field $F$. Let $I$ be the ideal of $F\left[X_{1}, X_{2}, \ldots\right]$ generated by the set

$$
\left\{X_{i} X_{j} X_{k}, X_{n} X_{n+1}-X_{1} X_{2}, X_{l} X_{m}|i, j, k, l, m, n \in \mathbb{N},| l-m \mid \neq 1\right\}
$$

and $R=F\left[X_{1}, X_{2}, \ldots\right] / I$. Rizvi constructed this example [11, p. 440] which, as indicated in [3, Examples 18.27], is a (commutative) local semiprimary continuous ring which is not self-injective. This ring satisfies the hypotheses of Corollary 2.7, but not those of Corollary 2.8 (as it is not right PF; see also Example (v) below). Thus both corollaries extend in different ways Osofsky's theorem which asserts that a right injective cogenerator ring is semiperfect and has finite essential socle.

On the other hand, this ring also provides an example which shows that the rings $R$ that satisfy the hypotheses of Corollary 2.7 (and hence those of Theorem 2.5) need not have the property that cyclic submodules of $E\left(R_{R}\right)$ are (essentially) embeddable in projective modules which, together with $|\Omega(R)| \leq|C(R)|$, also implies that $R_{R}$ cogenerates the simple right $R$-modules and has finite essential socle, as was shown in [6, Theorem 3.1]. Indeed, denoting $x_{n}=X_{n}+I \in R$ and letting $K$ be the ideal $K=\left\langle x_{2}, x_{3}, \ldots\right\rangle$, we see that, since $J=\left\langle x_{1}, x_{2}, \ldots\right\rangle$ is the only proper ideal containing $K$, the cyclic $R$-module $R / K$ has simple essential socle and hence embeds in $E(R)$ (as $\operatorname{Soc}(R)=J^{2}=\left\langle x_{1} x_{2}\right\rangle$ is essential and simple). However, it is 
readily seen that $K$ is not an annihilator ideal and hence $R / K$ does not embed in a projective module.

(v) The rings that satisfy the hypotheses of Corollary 2.9 need not be QF (compare with [8, Corollary 9] where, instead of $R$ being right CS, the projectivity of $E\left(R_{R}\right)$ was assumed). Indeed, any non QF ring with a unique finite composition series of right ideals (right uniserial ring) provides an example of this assertion. Observe that these rings also satisfy the hypotheses of Corollary 2.7 but not those of Corollary 2.8 .

\section{REFERENCES}

1. J. E. Björk, Radical properties of perfect modules, J. Reine Angew. Math. 245 (1972), 78-86. MR 47:1864

2. A. W. Chatters and C. R. Hajarnavis, Rings in which every complement right ideal is a direct summand, Quart. J. Math. 28 (1977), 61-80. MR 55:10519

3. Nguyen V. Dung, Dinh V. Huynh, P. F. Smith, and R. Wisbauer, Extending modules, Longman, Harlow, 1994. MR 96f: 16008

4. C. Faith, Algebra II Ring Theory, Springer-Verlag, Berlin and New York, 1976. MR 55:383

5. C. Faith, Embedding modules in projectives. A report on a problem, Lecture Notes in Math., vol. 951, Springer-Verlag, Berlin and New York, 1982, pp. 21-40. MR 84i:16001

6. J. L. Gómez Pardo and P. A. Guil Asensio, Essential embedding of cyclic modules in projectives, Trans. Amer. Math. Soc., to appear.

7. K. R. Goodearl, Von Neumann Regular Rings, Pitman, London, 1979. MR 80e:16011

8. P. Menal, On the endomorphism ring of a free module, Publ. Mat. Univ. Autonoma Barcelona 27 (1983), 141-154. MR 86g:16046

9. B. L. Osofsky, A generalization of Quasi-Frobenius rings, J. Algebra 4 (1966), 373-387. MR 34:4305

10. J. Rada and M. Saorín, On semiregular rings whose finitely generated modules embed in free modules, Canad. Math. Bull., to appear.

11. S. T. Rizvi, Commutative rings for which every continuous module is quasi-injective, Arch. Math. 50 (1988), 435-442. MR 89e:13009

12. B. Stenström, Rings of Quotients, Springer-Verlag, Berlin and New York, 1975.

13. R. B. Warfield, Jr., Serial rings and finitely presented modules, J. Algebra 37 (1975), 187-222. MR 53:5663

Departamento de Alxebra, Universidade de Santiago, 15771 Santiago de Compostela, SPAIN

E-mail address: pardo@zmat.usc.es

Departamento de Matematicas, Universidad de Murcia, 30100 Espinardo, Murcia, SPAIN

E-mail address: paguil@fcu.um.es 